BULLETIN OF THE

AMERICAN MATHEMATICAL SOCIETY

Volume 77, Number 1, January 1971

\title{
THE SUMMER MEETING IN LARAMIE
}

The seventy-fifth summer meeting of the American Mathematical Society was held in Laramie, Wyoming, from Tuesday through Friday, August 25-28, 1970. All sessions were held on the campus of the University of Wyoming. There were 928 persons in attendance, including 613 members of the Society. This meeting of the Society was held in conjunction with meetings of the Mathematical Association of America, the Institute of Mathematical Statistics, and $\mathrm{Pi} \mathrm{Mu}$ Epsilon.

The Colloquium Lectures were given by Professor R. H. Bing of the University of Wisconsin. The title of this series of lectures was Topology of 3-manifolds. Professor Bing was introduced by Professors V. L. Klee, C. E. Burgess, S. S. Cairns, and W. N. Smith.

By invitation of the Committee to Select Hour Speakers for Annual and Summer Meetings, there were two invited addresses. Professor Patrick P. Billingsley of the University of Chicago presented a lecture entitled Some probability results connected with Diophantine approximation. He was introduced by Professor Murray Rosenblatt. Professor Srinivasa S. R. Varadhan of the Courant Institute of Mathematical Sciences, New York University lectured on Diffusion processes: a martingale approach. The introduction of Professor Varadhan was made by Professor L. H. Koopmans.

There were nineteen sessions for the presentation of contributed papers. The following persons presided at these sessions: J. R. Edwards, T. L. Jenkins, D. C. Kent, J. E. Kirk, Jr., Guenter Krause, H. E. Lacey, R. T. Moore, J. A. Morrison, R. J. Oberg, A. D. Porter, B. G. Roth, D. E. Sanderson, E. C. Schlessinger, R. E. Smithson, A. K. Snyder, J. W. Van Ness, V. J. Varineau, G. F. Webb, and J. H. Wells.

The Council met on August 25, 1970, in the Rendezvous Room of Washakie Center at the University of Wyoming at 5:00 P.M. Professor J. T. Schwartz presided.

The Council received the minutes of Business by Mail dated July 31, 1970.

The Secretary announced the election of the following 267 persons to ordinary membership:

Abdeljaquad, Mahdi M., 6215 N.E. Radford Drive, Seattle, Washington 98115;

Abikoff, William H., 46 Garden Place, Brooklyn, New York 11201;

Adler, Leonda S., Sir George Williams University, Montreal, Quebec, Canada; 
Akers, David W., 1604 South Cherry Street, Olympia, Washington 98501;

Anand, Kailash Kumari, Sir George Williams University, Montreal, Quebec, Canada; Argyropoulos, Symeon D., 5145 Yarmouth Avenue, Apt. 8, Encino, California 91316; Asner, Bernard A., Jr., 2717 Pennington Street, Irving, Texas 75060;

Astor, Peter H., North Carolina State University;

Axmacher, Fred H. A. T., P. O. Box 3054, Eugene, Oregon 94703;

Bahnemann, David W., Northwest Missouri State College;

Baird, Leemon C., 704 Redbud Hill, Bloomington, Indiana 47401;

Baker, John A., University of Waterloo;

Baron, Celia M., Sir George Williams University, Montreal, Quebec, Canada;

Benson, David B., University of North Carolina, Chapel Hill;

Benton, Sister Thomas Aquinas, Our Lady of Holy Cross College;

Berezanskii, Jurii M., Ukrainian Academy, Kiev, U.S.S.R.;

Bergum, Gerald E., Gonzaga University;

Bernstein, Robert D., 14014 Panay Way, Marina del Ray, California 90291;

Berry, John W., Box 398, Notre Dame, Indiana 46556;

Bode, Joseph J., University of South Carolina;

Bolen, David W., Jr., Virginia Military Institute;

Borah, Bolindra N., A \& T State University;

Bragg, Lincoln E., University of Kentucky;

Brauchli, Hans T., 4315 Richardson Drive, N.W. Huntsville, Alabama 35805;

Bristol, Edgar H., 6 Maple Place, Foxboro, Massachusetts 02035;

Britton, William J., 1702 Meadow Street, Philadelphia, Pennsylvania 19124;

Brockett, Roger W., 5 Blinn Road, Lexington, Massachusetts 02173;

Brody, Jozef, 4200 Bourret Avenue, Montreal 249, Quebec, Canada;

Brooks, Barbara J., Bellcomm, Incorporated;

Brungs, Hans H., University of Alberta;

Buchmueller, Donald P., St. Bonaventure University;

Buttelmann, H. William, University of North Carolina;

Buraczewski, Adam, University of Science and Technology, Kumasi, Ghana;

Byers, Victor, Sir George Williams University, Montreal, Quebec, Canada;

Callegari, Andrew J., New York University, Courant Institute;

Cantor, Alan B., University of South Carolina;

Carlson, John R., 828 Maria Avenue, Spring Valley, California 92077;

Carmi, Gideon S., 640 W. 207 Street, New York, New York 10034;

Carvell, Robert, Jr., 1015 North Ravinia Drive, Dallas, Texas 75211;

Chang, Fu-Wu, 2262 Oakdale Street, Pasadena, California 91107;

Chiang, Min-Suei, Automatic Electric Laboratories, Incorporated;

Child, James D., 5700 Winton Road, Cincinnati, Ohio 45232;

Cho, Choong Yun, 5813 Ashfield Road, Alexandria, Virginia 22310;

Chorneyko, Ihor Z., McMaster University;

Chotlos, Bay I., Butler University;

Chrysovergis, Anthony, Sir George Williams University, Montreal, Quebec, Canada;

Cobb, Gary W., 4706 Placid Place, Austin, Texas 78731;

Cohen, Irving A., 2842 Sydney Street, Jacksonville, Florida 32205;

Cook, Frederick L., 1206 Kennamer Drive, S.E., Huntsville, Alabama 35801;

Coughlin, Raymond F., Loyola University;

Covitz, Howard H., Dalhousie University, Halifax, Nova Scotia, Canada;

Cramton, Thomas J., Purdue University;

Crossnoe, Marvin R., 5512-29th Drive, Lubbock, Texas 79407; 
Daley, Robert P., Carnegie-Mellon University;

Dana, Martin P., Washington State University;

Daskam, Robert A., Box 13281, Phoenix, Arizona 85002;

Depue, Bill E., Northop Institute of Technology;

Detemple, Duane W., Stanford University;

Dolney, Debra L., 110 Adriatic Avenue, Tampa, Florida 33606;

Drake, Stephen D., Box 277, Wurtsmith Memorial Schools, Clark AFB, Philippines, APO San Francisco, California 96274;

Dzabrailor, Allahveran D., Vavila Street, 42 USSR Moscow;

Ecklund, Earl F., Jr., Washington State University;

Elsey, William H., III, 2503 W. Garvey Avenue, \#35, Alhambra, California 91803;

Ettrick, Marco A., 1231 Lincoln Place, Apt. \#1, Brooklyn, New York 11213;

Ewing, Donald R., Environmental Science Services Administration, ERL, ITS, Room 2119, Boulder, Colorado 80302;

Fairweather, Graeme, Rice University;

Fajtlowicz, Siemion, 4246 Ridge Lea Road, Buffalo, New York 14226;

Feldstein, Philip, Fairleigh Dickinson University;

Feuer, Richard D., 270 West End Avenue, New York, New York 10023;

Finkelstein, Sheldon J., New York University, Courant Institute;

Fortson, Noble G., St. Mary's University;

Fox, Abraham S., Sir George Williams University, Montreal, Quebec, Canada;

Fox, Mary E., 300 Howard Avenue, Staten Island, New York 10301;

Frawley, William J., Schlumberger-Dahl Research Center, Ridgefield, Connecticut; Fremon, Richard L., Wayne State University;

Gangler, Joseph M., Jr., 327 Kings Highway, North Haven, Connecticut 06473;

Gallagher, Vernon L., 606 North Tenth Street, Bismarck, North Dakota 58501;

Gauntt, Robert J., California State College, Fullerton;

Giacaglia, G. E. O., University of Texas;

Glaser, Frank, University of California, Riverside;

Goldberg, Charles H., Trenton State College;

Goldfarb, Donald, 541 East 20th Street, Apt. \#8H, New York, New York 10010;

Grant, Kerry E., Southern Connecticut State College;

Grassmann, Eckhard G., University of Calgary;

Grefsrud, Gary W., Montana State University;

Griffiths, David F., New York University, Courant Institute;

Gruenhage, Gary F., University of California, Davis;

Gupta, Prahlad R., Aggarwal Company, Kurali (Punjab), India;

Haley, David R., 4800 Berwyn HS. Road, Apt. 606, College Park, Maryland 20740;

Halpern, Fred, Rider College;

Hamilton, Morris L., St. Andrews Presbyterian College;

Hammer, Peter L., Technion-Israel Institute of Technology, Haifa, Israel;

Hartfiel, Darald J., Texas A \& M University;

Harvey, John C. J., Jr., Montana State University;

Hayes, James C., 1361 Barsalou, Chamble, Quebec, Canada;

Head, James C., Simpson College;

Heath, Michael T., Union Carbide Corporation, P. O. Box P, Oak Ridge, Tennessee 37830;

Hejhal, Dennis A., University of Chicago;

Hemstead, Polly M., 256 Shipherd Circle, Oberlin, Ohio 44074;

Herr, David G., Duke University;

Herscovics, Nicolas, Sir George Williams University, Montreal, Quebec, Canada; 
Hibbs, Edwin B., Jr., 6109 Calmont, Fort Worth, Texas 11230;

Hill, Nancy E., 1326 Potomac School Road, McLean, Virginia 22101;

Hochberg, Murray, 730 Avenue L, Brooklyn, New York 11230;

Hofmann, Roseanne S., 1921 W. Olney Avenue, Philadelphia, Pennsylvania 19141;

Hotzel, Eckehart, McMaster University;

Howell, Leonard R., Jr., 1615 Southlawn Avenue, Fairborn, Ohio 45324;

Huebener, Mary J., 1718 Tuxworth Avenue, Cincinnati, Ohio 45238;

Hulme, Bernie Lee, 8115 Palo Duro, N.E., Albuquerque, New Mexico 87110;

Hummel, Kenneth E., Trinity University;

Hunt, Charles, Faculte des Sciences, Parc Valrose, (06), Nice, France;

Iskander, Awad A., American University of Beirut, Beirut, Lebanon;

Jacobs, Sidney, CUNY Graduate Center, 33 West 42nd Street, New York, New York 10036;

Jech, Thomas J., State University of New York, Buffalo;

Jones, Charles V., Western Illinois University;

Jones, Glenda M., 310 East Street, Apt. 3E, Madison, Indiana 47250;

Johnson, Howard C., 7642 S. Dante Avenue, Chicago, Illinois 60619;

Johnson, Robert W., Lehigh University;

Jung, Fuanglada R., 1021 Garden Way, Manhattan, Kansas 66502;

Kasprzak, Robert J., 2001 Prast Boulevard, South Bend, Indiana 46628;

Kine, Linda A., 20 Southern Way, Princeton, New Jersey 08540;

Klasa, Jacqueline, Northern Illinois University;

Knepple, George H., Box 804, Butler, New Jersey 07405;

Ko, Kan-Kwai, 183 King's Road, Flat E., 10th Floor, Hong Kong;

Kroll, Paul, 27 North 8 Street, Paterson, New Jersey 07522;

Kunzle, Hans P., University of California, Berkeley;

Labbe, Marcel A., University of Pittsburgh;

Labute, Tamara, Marianopolis College;

Lachtman, Mark E., University of Maryland;

Larson, Roland E., University of Colorado;

Leach, Ronald J., Howard University;

Lee, Francis R., 22 Huntington Street, Apt. 406, Hartford, Connecticut 06105;

Lee, Soo M., Taejon Presbyterian College, Chung-Nam, Korea;

Legge, John W., University of Oklahoma;

Lehpamer, Philip J., 1641 Balmoral, Westchester, Illinois 60153;

Leonard, Stephen B., Clarkson College of Technology;

Levine, Arnold, Tulane University;

Levine, Jeffrey M., Monmouth College;

Lin, Jer-Yih, Fayetteville State University;

Lin, Wen-Kang, 1408, 3463 St. Famille, Montreal 130, Quebec, Canada;

Loomis, Harlan W., U. S. Naval Weapons Laboratories, Dahlgren, Virginia;

Lowrie, Walter B., 15612 Patronella Avenue, Gardena, California 90249;

Lutchansky, Leo, Jr., P. O. Box 459, Nome, Alaska 99762;

Luther, Herbert A., Texas A \& M University;

Lyon, Andrew C., Rutgers University;

Mace, Vincent G., Jr., University of Washington;

Macgibbon, Brenda, McGill University;

Malik, Mohd A., Sir George Williams University, Montreal, Quebec, Canada;

Malviya, Banshid, McMaster University;

Maneki, Alfred P., North Dakota State University;

Manoharan, Alagesvaran C., Oklahoma City University; 
Marchetta, Sister Joan, College of St. Francis;

Martin, Dennis S., State University of New York, Brockport;

Martin, Robert C., Prentice-Hall, Englewood Cliffs, New Jersey;

Martin, Robert P., 8830 Hunting Lane, Laurel, Maryland 20810;

Mayne, David C., U. S. Naval Academy, Annapolis, Maryland 21402;

McGivney, Raymond J., Lafayette College;

McGlinn, Robert J., Southern Illinois University;

McGrath, Gary L., 527 West 5th Street, Winona, Minnesota 55987;

Meronk, David B., Bowling Green State University;

Meyrowitz, Alan L., University of Maryland;

Miletta, Peter D., Box 367, Norwich, Vermont 05055;

Miller, Michael D., University of California, Los Angeles;

Miller, Wayne L., Jr., 5404 Lorraine, Baytown, Texas 77520;

Minda, Carl D., University of California, San Diego, La Jolla;

Misra, Baidyanath, University of Rochester;

Mittag, Barry B., Brooklyn College;

Monaco, Fred R., 613 E. Queen Street, Inglewood, California 90301;

Montgomery, Ann, 500 W. Main Street, Apt. 24, Endicott, New York 13760;

Morgan, Alexander P., 284 Willow Street, New Haven, Connecticut 06511;

Morrison, Louise S., 2646 Fairway Avenue, South, St. Petersburg, Florida 33712;

Mucha, Karl S., Lot \#3, University Mobile Courts, Ruston, Louisiana 71270;

Muecke, Herbert O., Sam Houston State University;

Mueller, Ursula, Pennsylvania State University;

Mulvihill, Gail P., University of Massachusetts;

Murty, Tadepallis, Marine Sciences Branch, Department E.M.R., 615 Booth Street, Ottawa-4, Canada;

Namkoong, Kyu-Oh, State University College, Plattsburgh;

Naparsted, Abraham, State University of New York, Buffalo;

Natarajan, Subrahmanya, Florida State University;

O'Brien, Joseph N., Mankato State College;

Ogden, Nancy C., 983 Sylvania Drive, Dallas, Texas 75218;

Oliva, Frank A., II, 3525 Felton Street, San Diego, California 92104;

Olmstead, William E., Northwestern University;

Orton, Marion, 612 South Knightridge Road, Apt. 38, Bloomington, Indiana 47401;

Padia, William L., NAVFAC, Midway Island, Box 100, FPO, San Francisco 96614;

Park, Sehie, College of Liberal Arts and Sciences, Seoul National University, Seoul, Korea;

Paulowich, David G., Apt. 32, 6058 Pepperell Street, Halifax, Nova Scotia, Canada;

Perez, Lino M., P. O. Box 1912, Las Vegas, Nevada;

Pessin, Arthur L., Rider College;

Petrakopoulos, Nikitas L., University of Wisconsin, Green Bay;

Petty, Joe V., Texas Christian University;

Phan, Kok W., University of Notre Dame;

Pierce, Sam, University of California, Los Angeles;

Planchard, Jacques M., Electrite de France, 17 Avenue de la Liberation, 92 Clamart, France;

Poorman, Alan G., Ashland College, Ashland, Ohio 44805;

Prolla, Joao B., University of Rochester;

Pupko, Irving B., Central Michigan University;

Purifoy, Jesse A., 49 South Century Street, Memphis, Tennessee 38111; 
Ratchford, Mary A., Mount Saint Mary College;

Ray, Clifford H., Petty Geophysical Engineering Company, Tower Life Building, San Antonio, Texas 78205;

Reeves, Harry L., 6585 Prospect Street, San Jose, California 95129;

Reiser, Eugene J., 124 Oakville Drive, Pittsburgh, Pennsylvania 15220;

Richards, John F., 3226 Hartzell Street, Evanston, Illinois 60201;

Richter, Michael M., University of Texas;

Roberson, Fred A., Florida State University;

Rosich, Daniel, 1651 Metropolitian Avenue, \#2B, New York, New York 10462;

Roth, H. Daniel, Smithsonian Institution;

Ryan, Barbara F., Pennsylvania State University;

Sabel, Sister Clara Ann, Spalding College;

Saeks, Richard, University of Notre Dame;

Saini, Girdhari L., University of Saskatchewan;

Salam, Dianne M. W. T., 623 Hill Street, Highland Park, Illinois 60035;

Santiago, Armando, University of Puerto Rico;

Saxon, Stephen A., University of Florida;

Schlais, Harold E., Virginia Military Institute;

Schneider, Leo J., 18100 Weston Road, Cleveland, Ohio 44121;

Scruggs, William M., College of Southern Idaho;

Senez, John A., Sir George Williams University, Montreal, Quebec, Canada;

Sergent, Ernest, Jr., 1630 Erato Street, New Orleans, Louisiana 70130;

Shelupsky, David I., City College of New York, New York, New York 10031;

Shubsda, Stanley R., Jr., North Dakota State University;

Shuck, John W., University of Michigan;

Simanaitis, Dennis J., College of the Virgin Islands;

Simon, Barry M., Princeton University;

Smith, Norman E., 35 Summerhill Avenue, Valois, Quebec, Canada;

Smith, James D., Texas Christian University;

Smullyan, Raymond M., Herbert H. Lehman College (CUNY);

Srivastava, Jagdish N., Colorado State University;

Stack, Donald R., Ashdown Graduate House, Room 103 A, 305 Memorial Drive,

Cambridge, Massachusetts 02139;

Stark, Richard K., Route 3, Box 92, Gainesville, Florida 32601;

Stein, Barry N., Bronx Community College;

Stern, Charles M., III, Skidmore College;

Stux, Ivan E., Courant Institute of Mathematical Sciences;

Suffel, Charles L., 19-62 69th Avenue, Middle Village, New York 11379;

Sullivan, Michael E., 2021 Westcreek Lane, 82-B, Houston, Texas 77027;

Suryanarayana, Kuchibhotla V., Fayetteville State University;

Suter, Ueli, University of British Columbia, Vancouver 8, B.C., Canada;

Swenson, Carl E., Washington State University;

Swenson, Eva V., University of Toronto;

Symonds, Robin G., Trinity College;

Tamassy, Lajos, University of Debrecen, Debrecen, Hungary;

Tavernini, Lucio, Indiana University;

Teeters, Joseph L., Wisconsin State University;

Tips, James H., Jr., 707 Jackson-Keller Road, San Antonio, Texas 78216;

Tomkins, Robert J., University of Saskatchewan, Regina, Saskatchewan, Canada;

Upatisringa, Visutdhi, Humboldt State College; 
Updegraph, Charles, International Textbook Company;

Wakfer, Dagny, Ryerson Polytechnical Institute;

Walker, James W., Georgia Institute of Technology;

Walker, Sue A., Thomas J. Watson Research Center;

Washburn, Robert M., Southern Connecticut State College;

Wasileski, John S., Texas A \& M University;

Weinstein, Michael L., 3500 S.W. 24th Avenue, Gainesville, Florida 32601;

Welna, Cecilia, 176 Smith Street, New Britain, Connecticut 06053;

Wenzel, Gunter H., Queen's University;

Whitley, William G., University of Houston;

Wilkerson, Ralph W., Southern Illinois University;

Wilkins, Michael G., University of Virginia;

Willard, Earl R., Marietta College;

Williams, Scott W., Pennsylvania State University;

Wood, Dick A., Seattle Pacific College;

Wooster, Kenneth, State University College;

Wong, Cecile M., University of Montreal;

Wong, Roderick S. C., University of Manitoba;

Yu, Chung-Ling, Florida State University;

Zaki, Muhammad, Sir George Williams University, Montreal, Quebec, Canada.

It was announced that the following persons have been elected to membership in the Society in accordance with various reciprocity agreements:

Australian Mathematical Society: George Ivanov, Sidney A. Morris, Kenneth R. Pearson, Neil H. Williams.

Dansk Matematisk Forening: Hans J. Munkholm.

Deutsche Mathematiker Vereinigung e. V.: Herbert Amann, Walter A. Benz, KlausJurgen Scheele, Peter A. Thedy, Hans J. Vollrath.

Edinburgh Mathematical Society: David A. Brannan, Alan Jeffrey, Ian M. Michael.

Gesellschaft Fur Angewandte Mathematik Und Mechanik: Gerhard H. Opfer.

Indian Mathematical Society: Shashi P. Arya, Jagat N. Kapur, K. Chandrasekhara Rao.

Israel Mathematical Union: Mordechai Lewin, Abselom Ram.

London Mathematical Society: Simon J. Bernau, Brian W. Conolly, Frank R. Keogh, Millu Rosenblatt-Roth.

Mathematical Society of Japan: Fujiwara Daisuke, Soji Kaneyuki, Okumura Masafumi, Iino Riichi, Toshihiko Yamada.

Real Sociedad Mathematica Espanola: Gonzalo G. Lopez de Heredia, Jose L. Viviente.

Schweizerische Mathematische Gesellschaft: Emil R. Brandli, Renzo Cairoli, Peter Hess, Hans M. Reimann.

Sociedade De Mathematica De Sao Paulo: Odelar L. Linhares.

Societe Mathematique de Belgique: Jean J. Drabbe, Guy G. J. Noel.

Societe Mathematique de France: Georges C. Bohnke, Doan K. Bui, Yvonne Choquet-Bruhat, Julien Constantin, Jean-Michel Kantor, Thanh V. Nguyen, Antoine V. Visconti.

Svenska Matematikersamfundet: Ingemar L. Wik. 
Unione Matematica Italiana: Orazio Arena, Piero Bassanini, Massimo Fure, Emilio Gagliardo, Paolo Manselli.

Wiskundig Genootschap Te Amsterdam: Willem J. Blok, Willy G. van Hoorn, Eduardus Jager.

The following persons have been elected to membership upon nomination of institutional members as indicated:

University of Alabama, Birmingham: John P. Anderson, William H. Campbell, Karl R. Klose, Arthur C. Segal, Loy O. Vaughan, Jr., Patricia L. Weaver.

University of Alberta: Kasra Afsarinejad, James E. Chase, Sundaram Easwaran, Ramachandra Gopalan, Harry R. Henshaw, Mourad E. Ismail, Himatlal P. Jagani, Zia E. Khursheed, Kenneth G. Logan, Chung-Ping May, Michael M. Parmenter, Kar P. Shum, John N. Vardalas.

Amherst College: David M. Sanger.

Antioch College: Warren Z. Watson.

Arizona State University: Dennis N. Barr, Harold Bowman, David E. Brown, Garret E. Sobczyk, John A. Williams.

University of Arizona: Clifton B. Suitt, Nancy Tomlinson, David W. Zachmann.

University of Arkansas: Billy L. Ashmore, Earl E. McGehee, Jr.

Boise State College: Melvin L. Ott, Frank H. Smartt.

Bowling Green State University: Kwon-sang Chui, Vinit B. Khera, Shyun-Herng Tarn.

Brandeis University: Martin E. Flashman, Allan J. Gottlieb, Mauricio Gutierrez, Keith W. Harbaugh, William A. Teter.

University of Bridgeport: Daniel P. Doud, Milan E. Konopa, Jeffrey S. Leon, Gerald R. Martin, Valluru B. Rao, David A. Sibley.

University of British Columbia: Siow-Leong Ang, Richard A. Body, Albert G. Buckley, Derek Chambers, Dien-Wen Chen, Gregory V. Dropkin, David A. Fournier, Murray A. Jorgensen, Jagmohan Kapoor, Che B. Lam, Cheng-Ming Lee, Ezzat S. Noussair, Roy C. Snell, Duncan Suttles.

Brown University: Alan M. Baum, Paul Bourassa, John Casey, Tjee H. Chong, Richard S. Gavlak, Burton F. Gischner, Robert T. Glassey, Antonio F. Ize, Teisnke Ito, Ti-Jeun Kao, Robert E. Kowalczk, James C. Michener, Thomas G. Richardson, Tryfan G. Rogers, Tunglu Wang, Ronald J. Wojcik, Sr., Joel L. Wolf.

University of Calgary: Miloslav Nosal, Jon G. Rokne.

California Institute of Technology: Kwang-Nan Chow, Ralph S. Freese, Leonard J. Gray, Gregory N. Kourilsky.

California State College, Dominguez Hills: William L. Armacost, Stephen C. King.

California State College, Fullerton: Gloria G. Castellanos, Pasquale Francomano, David B. Surowski.

California State College, Long Beach: Howard Schwartz, Joseph J. Sroka, Kelly D. Tuell.

California State College, Los Angeles: Aaron F. Braziel, Leonard H. T. Dixon.

University of California, Berkeley: John P. Alexander, Roger K. Alexander, Victor M. Amezcua-Raz, Sara S. Baase, Ron C. Blei, Daniel L. Chester, Graciela Chichilnisky, Rolando A. Danao, Robert W. DeJean, Leonard A. Fellman, Barry S. Fogel, Steven R. Givant, James N. Hagler, Robert C. High, Gene R. Hall, David S. Johnson, Richard E. Ladner, Michael Legendre, Richard I. 
Loebl, Brian G. Luyt, Eduardo J. Mazzini, James R. Otto, Enrique A. Planchart, Simon R. Quint, William G. Reese, Luis Shein, Herbert B. Shulman, Sandor H. Straus, Rodham E. Tulloss, James G. Williams, King C. Yu, Fred M. Zlotnick.

University of California, Davis: Ray G. McAfee, Jr., Michael J. Zimmermann.

University of California, Irvine: John E. Kerlin, Jr., Anthony G. Mucci, Howard A. Seid.

University of California, Los Angeles: Marel K. Bates, Chi-Lung Chang, Roger M. Dell, James M. Edmondson, Bjorn O. Friberg, Douglas Fink, Don Krakowski, Steven R. Lay, Hadi J. Mustafa, Robert E. Park, Chu-Chin Peng, N. B. Perceval-Price, Alan Radnitz, Rolf M. Range, Ronald J. Rudman, Francisco J. Samaniegs, Joel L. Schiff.

University of California, Riverside: George R. Gordh, Jr., Stephen J. Wolf, Misha Zafran.

University of California, San Diego: George D. Parker.

Calvin College: Janice F. Baker, William B. Grasman, Roger D. Konyndyk.

Carnegie-Mellon University: David H. Buckland, Isaac E. Leonard, Peter J. Nyikos, Marion L. Oliver, William T. Patula, Barbara S. Thomas.

Central Michigan University: Theodore H. Hosner, David L. Lake.

Chicago State College: Don D. Bunt, James P. Cottingham, James P. Skinner.

University of Chicago: Celio W. Manzi, James E. Archer, Bruce E. Beeken, ChiuChun Chang, Frederick R. Cohen, Gerald J. Decker, Adilsoiv Goncalves, Alvaro Gonzalez Villalobos, Robert L. Griess, Jr., Alberto Guzman, Chat-Yin Ho, Richard N. Lyons, Ronald W. Ming, Stylianus Pichorides, Bruce A. Prekowitz, Judith D. Sally, Alberto Torchinsky, Liang-Chi Tsao, Adrian R. Wadsworth, Kai Wang, Theodore F. Zelman.

Brooklyn College, City University of New York: Joseph Chesir,

Herbert H. Lehman College, City University of New York: Robert T. Craig, Stephen F. Markstein.

Hunter College, City University of New York: Esther E. Cantor, Robert M. Carroll, Pearl Greenberger.

Queens College, City University of New York: Maury I. Horowitz.

Clark University: Robert W. Redding, Gerald Wildenberg.

Clemson University: Gordon L. Bailes, Jr., Marilyn J. Breen, Gerard P. Protomastro.

Colorado State University: Kathleen M. Timmer.

University of Colorado: John C. Adams, Raymond A. Arehart, David L. Carlson, Eloise H. Carlton, Richard S. Collier, Arlo D. Davis, Larry V. Ellis, Walter G. Kelley, Raj K. Markanda.

Columbia University: Seth R. Alpert, Erie V. Boorman, III, Evelyn J. Boorman, Kevin A. Broughan, Fu-Shih Cheng, Richard H. Frobose, Jr., George F. Gruenthal, Fu Yeh.

Concordia College: Donald Randall, Clifford O. Ranheim.

University of Connecticut: Dale T. Hoffman, Louis Mercuri, Richard I. Resch, Arsine Rustigian, Janet E. Teeguarden.

Dartmouth College: Michael J. Berg, Thomas H. Brylawski, David S. Cochran.

University of Dayton: James E. Felt, George J. Gipp, James A. Schoen.

University of Detroit: Robert J. D'Amico, Stephen M. Grimley, Thomas W. Klamo, Mary E. Kopytec, Sister Teresa Reid, Michael G. Ricci.

Duke University: Lee O. Hagglund, Ralph E. Johnson, David C. Kurtz, Larry D. Shatoff, Geoffrey R. Webb. 
East Carolina University: Nannie Lee W. Manning, Louise L. Williams.

East Texas State University: Farhad Aslan, Alton R. Goodard, William V. Underhill.

Eastern New Mexico University: Woodrow W. Overstreet, Jr.

Emory University: Phillip A. Hartman, James C. Kropa, Muril L. Robertson.

Florida Presbyterian College: Pablo Perhacs.

Florida State University: Eduardo R. Bastida, Robert W. Chestney, Anne P. Grams, Joseph F. Hoffmann, Kathleen B. Levitz, Elizabeth W. Moore, Michel F. Schexnayder, Brian J. Wesselink.

University of Florida: Stephen J. Maxwell, Desmond A. Robbie.

Franklin and Marshall College: Darrell E. Haile, Walter D. Mendenhall, Nelson E. Zindell.

Georgia Institute of Technology: David L. Lovelady.

Georgia State College: Ralph T. Bishop, James C. McGarry, T. David R. Neill.

University of Georgia: Frank L. Knowles, Richard H. Reese, Kosmo D. Tatalias.

Gustavus Adolphus College: Gwendolyn E. Moberg, Donn C. Sandell, Dennis Wellnitz.

Harvard University: Ellen D. Borie, John H. Coates, Pierre R. Deligne, Antoine L. Derighetti, Stephen T. Fisk, William J. Gordon, Finn F. Knudsen, Kenneth B. Kramer, Eric P. Kronstadt, Stacy G. Langton, Victor S. Miller, Charles B. Setzer, David J. Smith, Helen P. Wang, William F. Wilson, Charles L. Zucker.

University of Houston: Betty J. Hinman, Roberto A. Mena.

University of Idaho: David J. Ferguson.

University of Illinois: Eric M. Barton, Raymond E. Beasley, Istvan Beck, Richard S. Bukowski, Patrick D. Cassady, Christopher F. Cox, Peter R. Eiseman, James K. Finch, Eric Frankl, Masatoshi Fukushima, Charles R. Hadlock, Henry N. Hayhoe, Margaret E. Mann, William L. Perry, V. R. Gopala Rao, Ivan L. Reilly, William B. Stewart, Paul L. Strong, Joseph R. A. Strutt, Ramani Vasudevan, Subramanian Venkateswaran.

Illinois Institute of Technology: Stuart J. Meshboum.

Indiana University: Stephen Berman, Patrick M. Brown, Donald L. Curlovic, David A. Cusick, Robert A. Funk, Gerald N. Hile, Yang Ho, Kapil D. Joshi, Roger C. Lautzenheiser, Howard J. Marcum, Richard L. Marcuson, Samuel Masih, David L. Parker, Nand K. Sharma, Thottathil V. Varughese, Jay R. Walton, Robert E. Weber.

Iowa State University: James E. Brink, Pi-Chun Chuang, Elvin M. Eltze, Jack E. Girolo, Randal P. Miller, Marcus J. Saegrove.

University of Iowa: Daniel D. Anderson, Daniel J. Britten, Michael E. Detlefsen, Donald A. Myers, Dale A. Schoenefeld.

Johns Hopkins University: Ronald M. Benincasa, James M. D'Archangelo, Nishan K. Krikorian, Jr., Erika Orfield, Truman W. Prevatt, Bruce I. Powers, Joseph D. Schwartz, Irene Verner.

University of Kansas: Sheldon Adelberg, Martin F. Cheng, James C. Deel, Gerd H. Fricke, Donald E. Kruse, David C. Lantz, Christopher L. Samelson, James R. Schap, Louis A. Talman, David J. Thuente.

Kent State University: Gene R. Sheller, Tsu-Yang A. Wang.

University of Kentucky: Thomas J. Cheatham, Edson W. Ewing, Charles R. Hammons, James K. Harness, Richard W. Leggett, John K. Shaw, George F. Weida, Lynn R. Williams.

Knox College: Steven A. Harshbarger, Robert N. Solin, John C. Tripp.

Kutztown State College: Edward W. Evans, Kyu S. Park. 
Lehigh University: Marcia M. Shunta.

Lemoyne College: John J. King, Christopher B. Martin.

Long Island University: Jeffrey St. Goldstein, Doris J. Ruopp, Ira P. Stoler.

Louisiana State University: Richard M. Caron, Thomas A. Chapman, Lawrence A.

Couvillon, Donald M. Hill, Alvin E. Horne, James W. Lea Jr., Daniel R. Lewis, William J. Lewis, Edith A. McCharen, Virginia J. Pollard, David E. Rush, Carlos G. Spaht, II, William A. Vekovius.

Louisiana State University, New Orleans: Lonny C. Breaux, Elizabeth R. Carriere, Richard H. So.

Loyola College: John A. Gaynor, Anthony T. Valenti, Richard A. Wisniewski.

Loyola University, New Orleans: Gregory J. Reibenspies.

University of Maine: June E. Ackley, Elizabeth M. Ray, Alan D. Taylor.

Marquette University: Stephen L. Campbell, Ingrid Kosanetzky, Willard E. Lawrence.

University of Maryland: Nazar H. Abdelaziz, Daniel T. Adams, David A. Andress, Gregory L. Caucutt, Robert W. Clarke, Suellen Everhart, Robert J. Galia, Rao V. Govinparaju, Gilbert V. Jacobs, Marvin Kasper, Warren R. Luckner, James R. Miller, Jorge J. Moré, Rainer J. Nagel, Osborne C. Parchment, Ralph P. Pass, III, Ulf Schlotterbeck, Judith S. Sunley, Edwin E. Whitaker, Harry S. Wieand.

Massachusetts Institute of Technology: Mark J. Ablowitz, Jose L. Abreu, Tadatoshi Akiba, Kenneth S. Brown, Shu-Kwan Chan, Emmanuel F. Dror, Susan J. Friedlander, William H. Henneman, Siu-Ming Ho, Abel Klein, Jay J. Lagemann, Kenneth L. Lange, Russell L. Mallett, Robert Mandl, Alan S. Pollack, Hans A. Salomonsen, Jayant M. Shah, Richard A. Shore, Stephen W. Smoliar, Paul S. Wang, Walter J. Whitely, Edward B. Williams, Walter S. Wilson.

University of Massachusetts: Normand H. Cote, Alan Gorfin, Robert A. Haberstroh, Carlos S. Johnson, Jr., David L. Stenson.

McGill University: Elias Brettler, Anne E. Drinkwater, Robert E. Williams.

McMaster University: Walter M. Dubiel, Peter L. Wright.

University of Miami: James C. Burkett, Kenneth K. Lau, Gilbert F. Orr, Henrietta A. Presnell.

Michigan State University: Paul F. Murphy, Charles J. Parry, Joseph E. Quinn.

University of Michigan: Donald K. Blevins, Stephen T. Brewster, Ian M. Chiswell, John W. Dawson, Jr., Wayne C. Ellis, Per Hag, Włodzimierz Holsztynski, Richard J. Orr, Theodore T. Papas, David A. Perin, Martin Sommerfield, Thomas C. Spencer, Catherine E. Stahl, Robert M. Young.

Middleburg College: Jean S. Frederiksen, Wilfred B. Shepardson.

Millsaps College: Linda S. Dorsey, Michael D. Johnson, James T. Smith.

University of Minnesota: John T. Burns, Nancy M. Campbell, Raymond J. Collins, Ned. M. Gibbons, Wayne R. Jones, Chul E. Kim, Ronald E. Kutz, William K. Perrizo, Justin R. Peters, III, Douglas E. Polley, Koon-Yul Poon, Ronald D. Priebe, Philip J. Siller, James W. Willen.

University of Mississippi: Alvin F. Barr, Chester W. Richards, Howell W. Todd.

University of Missouri: Robert A. Chaffer, Thomas J. Miles, Steve E. Mosiman, Ronald L. Sinzdak.

University of Missouri, Kansas City: H. S. Gopalakrishna, Steve C. Hansen, James W. Walker.

University of Montreal: Serge Bissonnette, André Joyal, Michel Laramee, Pierre Laviolette, Loc Vo Ba, Edouard Wagneur.

University of Montana: Daniel L. Hansen, Bernard W. Irlbeck. 
Morehouse College: Sidney H. Hankerson, Clarance M. Myers.

Murray State College: Judith A. Carlisle, J. C. Hankins, Richard E. Smothermon. University of Nebraska: Monte B. Boisen, Jr., Randall K. Heckman, Charles T. Shipley.

Newark College of Engineering: Robert S. Pujat, Adrian L. Simons.

University of Nevada: Jerry I. Blair, Harvey W. Lambert, Steve T. Timko.

University of New Brunswick: Geraldine F. LeBlanc, David E. Morris, Michael J. Wood.

University of New Hampshire: Jiann-Jer Chen, Curtis P. Murley.

University of New Mexico: Vernon L. Bakke, Taen-Yu Dai, James O. Friel, Robert D. Russell, Barry Walter.

New York University: Laren C. Bruzzone, Jeffrey I. Caro, Kenneth C. Chen, David B. Cohen, Richard S. Ellis, Bjorn E. Engquist, Karen L. Ginsburg, Jerry R. Hobbs, David H. Hoitsma, Richard J. Jarvis, Nadav Liron, Melvin R. Mullin, Reinhard M. Olivier, Tunekiti Sirao, Andrew M. Soward, Nancy E. Zumoff.

University of North Carolina, Chapel Hill: Joseph W. Goldston, Suzanne L. Miller, Bruce L. Murrie, Walter J. Thomson, James M. Westall, Jr.

University of North Carolina, Charlotte: Thomas R. Lucas, Vicente N. Hernandez, Glenn R. Long, Fred Toney, Jr.

University of North Carolina, Greensboro: Elizabeth E. Bray, Dargan Frierson, Jr., Robert E. Michaud.

University of North Dakota: L. Thomas Ramsey, Dave R. Schmitz, John S. Steinberger, Jr.

North Texas State University: Willis L. Dooley, Don M. Keisler, Charles S. Stevens.

Northern Illinois University: William F. Lipman, Sean D. McCartan, Liam O'Carroll.

Northern Michigan University: Joseph B. Browne, Marion L. Ellerbruch, Susan M. Orhanen.

Northwestern University: Michael G. Barratt, James V. Blowers, Willy Brandal, Daniel R. Jurca, David G. Kostka, Gary S. Patrik, John H. Reeder, Robert W. Shurtleff, Carl P. Simon, Richard E. Snow, Leon C. Stecher, Donald W. Swanton, Paul A. Thomas, Hirosi Toda.

Norwich University: Joseph J. Heed, Donald E. Nevin, Gordon C. Smith.

Notre Dame University: Robert P. Boner, Chan-Nan Chang, Richard H. Escobales, David J. Hickman, Kenneth E. Martin, Randolph J. Ostlie, David H. Tracy, Tah-Zen Yuan.

Oakland University: Steven J. Takiff.

Ohio State University: Richard A. Bieberich, Dear R. Brown, Daniel A. Falk, Usha Sachdeva, Wilbur P. Veith, Thomas J. Woolley, Kenneth P. Yanosko.

Oklahoma State University: Melvyn W. Jeter, Glenda K. Owens.

University of Oklahoma: Larry F. Bennett, Clarence O. Durand, Philip J. Schapiro.

Old Dominion University: Gloria B. Barrett, Glenn R. Burdick, Stephen F. Fischer.

Olivet College: David M. Symonds.

Oregon State University: Roger L. Higdem, Jack E. Hofer, Ralph L. James, Edward M. Matzdorff, Thomas J. Mueller, Isamu Okajima, Nagendra N. Pandey.

University of Oregon: Bruce N. Braunsten, Steven E. Brown, Michael J. Gilpin, Hugh W. Goldhammer, Rita M. Hall, Rahim G. Karimpour, Robert A. Lindenberg, Lewis Lum, Johnathan Stafford, Brian M. Stecher.

Pace College: Louis J. Braun, Maureen C. Gallagher, Max P. Hoefer.

Pacific Lutheran University: David W. Kesler, Ingrid M. Knutzen, Stephen H. Mangelsen. 
Pennsylvania State University: Richard G. Rehberg, Kandiah Thanigasalam, Manfred Stoll, David E. Zitarelli.

University of Pennsylvania: Salvador D. Gigena.

Portland State College: Hwa-Lin V. Huang.

Princeton University: William E. Beckner, Marshall W. Buck, Tito R. Chavez, Eli N. Donkar, David M. Finkel, William D. Homer, II, Robert E. Knapp, YoKo Nakagawa, Charles Rockland, Kuang-yen Shih, Steven H. Weintraub.

University of Puerto Rico, Mayaguez: Rodriquez C. T. Bernier, Luis A. Osorio.

Purdue University: William J. Browning, Bang-Dar Cheng, Gene A. DeBoth, Christine A. Dombrowski, John A. Gosselin, David I. Heimann, Judith M. Hutchens, Mohan C. Joshi, Walter E. Maxey, Barbara B. Moore, Cecil P. Murray, Kevin M. O'Brien, Steven A. Pruess, William B. Ringland, Edward R. Rozema, Phillip H. Schmidt, Philip W. Smith, Rudy G. Svoboda, W. Scott Tamblyn, III, Harold D. Victory, Jr., Wo-Sang V. Young.

Rensselaer Polytechnic Institute: Paul J. Faix, Carolyn F. Klimek, William J. Steinmetz.

University of Rhode Island: James T. Lewis, A. N. Vijayadhwaja Rao.

Rice University: Robert Carmignani, John C. Martin.

University of Rochester: Kenneth A. Bergstresser, Jr., Allan M. Carlton, Melvyn B. Nathanson, Diane S. Radin, David L. Smallen.

Rockefeller University: Ulf $H$. Friedrichsdorf.

Rockford College: David A. Englund.

Sacramento State College: Fung-Chu Chen, Wade I. Jones.

St. Olaf College: Peter J. Munson, Marcia M. White, John D. Zicarelli.

Salem State College: Peter C. Wong.

San Diego State College: Jonathan G. Bell, Lee K. Byrom, Patrick A. Staley.

San Francisco State College: David L. DeGeorge, Wanda E. Miller, Robert B. Tieslau.

University of Saskatchewan, Regina: Michael A. Gates, Joseph Mogyorosy, Charles R. Seaman, Dale V. Thompson.

University of Saskatchewan, Saskatoon: John W. Stephenson, Kee-Leong Teo.

Simon Fraser University: Jorge A. DeCecco, Lance Gutteridge, Robert H. Redfield.

Smith College: Mei-Chin H. Ku.

University of South Carolina: Joseph E. Cicero, Simon C. Hsieh.

South Dakota School of Mines \& Technology: Jon O. Lehner.

University of Southern California: John D. Featherstone, Marilyn K. Oba, Bhalchandra B. Phadke, Edward G. Thurber, Zdenek Vorel, Thomas M. Wenska.

Southern Illinois University, Carbondale: Gerald A. Kraus.

Southern Illinois University, Edwardsville: Patricia E. Hicks, Thomas A. Kriz, Bonnie D. Rowland.

Southern Methodist University: John V. Beverstock, Mary C. Carter, Robert I. Mann.

Southwestern at Memphis: Charles L. Chandler, David C. McNeel, Elizabeth R. Ridings.

State University College, Plattsburgh: Deedrick P. Bertholf, Ronald F. Dufort, William P. Gibbons.

State University of New York, Binghamton: John H. Ying.

State University of New York, Buffalo: Jay H. Beder, Douglas R. Busch, Pui W. Chan, Pilar de la Torre, Leonard C. Gambler, Edwin T. Hoefer, Miroslav I. Klun, Isao Ozaki, Pey L. Pan, William C. Powell, T. K. Puttaswamy, Narayana G. 
Reddy, Steven G. Schlosser, T. R. Sundararaman, Hung P. Tan, Richard W. Yoh.

State University of New York, Stony Brook: Christine B. Beaucage, Richard W. Carey, Sang M. Kim, Subir Nanda.

Stephen F. Austin State University: William M. Peterman, Daniel A. Weaver.

Syracuse University: Gary M. Sampson.

Temple University: Loretta P. Campbell, Joel M. Recht, Paul D. Trembeth.

University of Tennessee: Jacob T. B. Beard, Jr., Thomas E. Hays, George W. Johnson, $H$. Allen Walker, James R. Wall.

Texas Christian University: Robert D. Carmitchel, David M. Henry, Donald F. Reynolds.

Texas A \& I University: Gary D. Middleton, Carletta Miller, Joyce M. Slough.

Texas A \& M University: Carolyn J. Slama, Jimmy L. Solomon.

University of Texas: Colin Bennett, Robert S. Boyer, David R. Kincaid, Gary R. McDonald, Robert C. Sharpley, Jerry C. Webb.

Texas Technological College: John C. Drummond, Jr.

Texas Technological University: George D. Poole, Everette D. Williams.

University of Toledo: Barbara J. Taylor.

University of Toronto: George J. Hariton, Ronald Jhu, Marta A. Kovarik, Zdislav V. Kovarik, Ronald R. Miller, Alfred Pietrowski, Leonidas Zafiriu.

Trenton State College: Edward J. Conjura, Nicholas N. Greenbaum, Robert M. Wharton.

Trinity College: Grant W. Branstator, Howard K. Gilbert.

Tufts University: Wen Mong Lee.

Union College: Robert W. Ackley, Ronald A. Wendoloski, Michael G. Yochmowitz.

University of Utah: Orville L. Bierman, Robert M. Harris, Max R. Lund, Keith B. Olson, Frederick Sipinen, Barbara A. Stewart.

Vanderbilt University: David E. Bell, Eleanor C. Smith.

Vassar College: Jack Prins.

University of Vermont: Michael V. Riley, Judith S. Walters.

Virginia Commonwealth University: Robert W. Murdock, Pratip N. Raychowdhury, David A. Schedler.

Virginia Polytechnic Institute: Chang-Ming Hsu.

University of Virginia: Keith R. Allen, Gerald M. Bryce, David E. Cooper, Victor W, Daniel, Wynne A. Guy, Jack P. Sanders, Richard E. Sours.

Villanova University: Sister Marguerite Bonner, Victor J. Costanzo, Robert D. Martin.

Wake Forest University: Glenda S. Angel.

Washington State University: David J. Smith.

Washington University: Vance Faber.

University of Washington: David E. Chalif, Jane E. Cope, John W. Donnelly, Timothy R. Eaton, Richard A. Erickson, Eric R. Halsey, John R. McMullen, Frank B. Miles, David F. Neu, Vaho Rebassoo, Joseph M. Rosenblatt, Ruth Rubinstein, Robert W. Shannon.

Wayne State University: John P. Beckwith, Dhandapani Kannan.

University of Waterloo: Alan A. Adamson, Forbes J. Burkowski, Václav Chvátal, Anton W. Colijn, James E. Totten, Mark S. Wolynetz.

Wellesley College: Claire L. Parkinson.

Wesleyan University: Robert B. Chapman, Jr., Kenneth N. Orbach.

West Georgia College: John W. Burtchaell, James E. Perry. 
Western Michigan University: Timothy B. Carroll, Paul E. Himelwright, III, Pascal D. Mubenga, George D. Reynolds, Arnold. H. Veldkamp.

West Virginia University: John D. Klemm.

Western Washington State College: Thomas J. Smircich.

Wheaton College: Candice C. Bryant, Donna E. Elton, Patricia D. Scully.

College of William \& Mary: Robert L. Anderson, David A. Dickey, Du-Shan Tsou.

Williams College: John C. Peinert.

University of Wisconsin: Christina A. Bahl, John W. Ballard, Michael P. Benson, Duane M. Broline, Alfred S. Cavaretta, Jr., Evans W. Cheeseman, Jr., Richard C. Detmer, George T. Diderrich, Jr., Diana Fields, Thomas H. Foregger, Dwight D. Freund, Sidney C. Garrison, Andrew M. Glass, Scott W. Jennings, Rosa B. Jones, Paul D. Kindschi, Clinton J. Kolaski, Philip C. Kutzko, John J. Libera, Jr., Arthur R. Lubin, Jitendra N. Manocha, Sherry L. Masters, Douglas B. McLeod, Robert W. Miller, James D. Newton, Yung S. Oh, Julius W. Overbeck, Jonathan T. Patten, Richard C. Randell, Franklin B. Richards, Gerhard X. Ritter, John A. Rosenberg, Sherwood D. Silliman, Richard M. Smith, Robin Soloway, Joze Vrabec, Peter Warren, Perry Williams, William J. Yoder, Sergio E. Zarantonello.

University of Wisconsin, Milwaukee: Richard J. Fleissner, Bruce W. Mielke, Leo G. Sobieski.

Yale University: James G. Arthur, Benjamin G. Cooper, Neal Felsinger, John C. Hemperly, Lowell E. Jones, James M. Mathews, Brian J. Parshall, Arnold K. Pizer, Richard D. Porter, Jr., James E. White.

Yeshiva University: Roger M. Aarons, Samuel Kohn, Mary Y. Shiffman, Morton Slater.

York University: Gordon M. Armstrong, Alan H. Mekler, Lawrence A. Swain.

The Secretary announced the election of the following organizations to institutional membership:

University of Alabama, Birmingham, Alabama

Eastern Montana College, Billings, Montana

George Mason College of the University of Virginia, Fairfax, Virginia

Kutztown State College, Kutztown, Pennsylvania

Loyola University, New Orleans, Louisiana

Miami University, Oxford, Ohio

Murray State University, Murray, Kentucky

University of North Carolina at Wilmington, Wilmington, North Carolina

Université de Québec á Chicoutimi, Chicoutimi, Québec, Canada

State University College, Plattsburg, New York

The Secretary recorded that the following persons represented the Society at various functions as noted:

Peter J. Kiernan at the inauguration of Frank N. Elliott as President of Rider College

Burrell W. Helton at the inauguration of Joe K. Menn as President of Texas Lutheran College

Richard F. Gustafson at the inauguration of Adolph Gustof Anderson as President of Hartwick College 
The President established Committees and added members to Committees as follows:

Committee to Select Hour Speakers for Southeastern Sectional Meetings: C. H. Edwards, Jr. and Trevor Evans

Steele Prize Committee: Felix Browder, S. S. Chern, Murray Gerstenhaber, Chairman; Edwin Hewitt, John W. Milnor, Abraham Seidenberg, I. M. Singer

The following persons have accepted invitations to present hour addresses at the times and places noted:

$\begin{array}{lll}\text { Ramesh Gangoli } & \text { Davis } & \text { April } 1970 \\ \text { P. Billingsley } & \text { Laramie } & \text { August } 1970 \\ \text { S. Varadhan } & \text { Laramie } & \text { August } 1970 \\ \text { Donald Ornstein } & \text { Pasadena } & \text { November 1970 } \\ \text { James Dugundji } & \text { Monterey } & \text { April 1971 } \\ \text { Andreano Garsia } & \text { Monterey } & \text { April 1971 } \\ \text { R. J. Blattner } & \text { Corvallis } & \text { June 1971 } \\ \text { Ky Fan } & \text { Corvallis } & \text { June 1971 } \\ \text { Harry Kesten } & \text { Atlantic City } & \text { January 1971 } \\ \text { Leopoldo Nachbin } & \text { Atlantic City } & \text { January 1971 } \\ \text { Daniel G. Quillen } & \text { Atlantic City } & \text { January 1971 } \\ \text { Dennis Sullivan } & \text { Atlantic City } & \text { January 1971 }\end{array}$

The report of the Nominating Committee consisting of Richard Brauer, Earl Coddington, Paul Halmos, Kenneth M. Hoffman, and Emery Thomas, Chairman, was accepted by the Council. It provided for the following nominations:

Trustee:

P. Bateman

Vice President (one to be elected):

H. Royden

E. Spanier

Secretary:

E. Pitcher

Treasurer:

W. T. Martin

Associate Secretary:

W. Gottschalk

O. Harrold

Bulletin Editorial Committee:

G.-C. Rota

Proceedings Editorial Committee:

F. Brauer

Colloquium Editorial Committee:

A. Calderón

Mathematical Reviews Editorial Committee: R. Pierce

Transactions and Memoirs Editorial Com-
mittee:
H. Kesten
D. S. Rim

Mathematical Surveys Editorial Committee: E. H. Brown, Jr. 
American Journal of Mathematics Representative:

H. Bass

Members-at-large of the Council

(five to be elected):

C. B. Bell, Jr.

G. F. Carrier

Morton L. Curtis

R. C. Kirby

W. C. Royster

Mary Ellen Rudin

D. E. Sarason

J. B. Serrin

E. M. Stein

S. E. Warschawski

The Council elected S. Orey and S. Warner to the Editorial Board of the Duke Journal. They elected M. Gerstenhaber and O. Harrold to represent the Society in Section A of the American Association for the Advancement of Science. They nominated P. Hilton and C. C. Lin to represent the Society in the Division of Mathematical Sciences of the National Research Council.

The Council received a report from the Committee of Visitors to Tulane University over the signatures of R. H. Bing and E. H. McShane. The Committee had been appointed at the suggestion of Tulane's President, Herbert E. Longenecker, to discuss matters of mutual interest. These arose in connection with the termination of employment of Dr. Edward L. Dubinsky at that university. The Council discharged the Committee with special thanks asking the Secretary to record that they recognized the difficult circumstances under which the information was gathered, the excellence of presentation of the report, and the service of the Committee to the Society. [The Secretary has a few copies of the report available for distribution on request.]

The meeting adjourned at 6:35 P.M.

The Business Meeting was convened by Professor Schwartz on August 28, 1970, in the Auditorium of the Arts and Science Building. Mr. Frank Mockler served as parliamentarian. Associate Secretary R. S. Pierce served as Acting Secretary.

The first order of business was the presentation of the first Steele Prize, in honor of George D. Birkoff, William Fogg Osgood, and William Caspar Graustein, to Professor Solomon Lefschetz for his paper, "A page of mathematical autobiography," published in the Bulletin of the American Mathematical Society, Vol. 74 (1968), pp. 854-879. 
Professor Lefschetz was unable to be present; at his request, his thanks were expressed by Professor Joseph P. LaSalle.

The report of the Secretary consisted of an account of several actions of the Council reported above and in volume 76 of this Bulletin, pp. 691-692.

The Business Meeting gave unanimous approval to an amendment to the By-laws whose effects are to make the Committee to Monitor Problems in Communications be a statutory committee, with members nominated by the nominating committee and elected by the membership; to make the chairman of the committee, elected by the committee, be a member of the Council; to make the current members of the appointed committee be the initial elected members with staggered terms; and to charge the committee with performanie of such tasks in the field of communication of mathematics as are assigned to it by the Council.

Formally, the amendment consisted of adding words (indicated in italics) and making a deletion (indicated by a strike-over) as follows:

Article III. Change the title.

Publications and Communications Committees

Article III, Section 1. Insert words.

There shall be nine Publications Committees, which shall consist of the nine Editorial Committees specified in Section 2 of this article and one Communications Committee.

Article III, Section 4. A new section.

There shall be a Communications Committee called the Committee to Monitor Problems in Communication.

Article III, Section 5. The old section 4 with words inserted.

The size of each Publication and Communications Committee shall be determined by the Council.

Article IV, Section 1. Insert words.

The Council shall consist of fifteen members at large and the following ex officio members: the officers of the Society specified in Article I, the members of the Publications Committee and the Chairman of the Communications Committees specified in Article III, etc.

Article VI, Section 3. Add a sentence to the duties of the Executive Director.

He shall be a voting member of the Committee to Monitor Problems in Communication but shall not be its chairman.

Article VII, Section 1. Insert words in sentence 2.

The term of office in case of members of the Publications and Communications Com- 
mittees (excepting the latter in 1970 through 1972) shall be . . . and three years for the remaining committees, except that when the size of a Publication or Communications Committee is changed, the Council may authorize the election of a member for a shorter term.

Article VII, Section 1. Add a sentence.

The members of a Communications Committee with terms ending on December 31 are designated as Past Members through February 10 of the following year.

Article VII, Section 1b. A new section.

For the years 1970 through 1972, the terms of the members of the Committee to Monitor Problems in Communication shall be as follows:

$\begin{array}{ll}\text { Alex Rosenberg } & 1970 \\ \text { Daniel Zelinsky } & 1970 \\ \text { Jim Douglas, Jr. } & 1971 \\ \text { Ivan Niven } & 1971 \\ \text { Frank Peterson } & 1972 \\ \text { Allen Shields } & 1972\end{array}$

On January 1, 1973, this section, as well as the parenthetical phrase "(excepting the latter in 1970 through 1972)" of Section 1 will be repealed.

Article VII, Section 2. Insert words in sentence 1.

The President elect, ...., the members of the Publications and Communications Committees ... shall be elected by written ballot.

Article VII, Section 2. Add a sentence.

Each Committee on Communications elects one of its members as chairman in a manner and for a term designated by the Council. Past Members as defined in Article VII may be authorized to vote in this election.

Article VII, Section 6. Insert words in sentence 5.

Such vacancies as may occur at any time in the group consisting of ... and the members of the Publications and Communications Committees shall be filled by the Council... .

Article VII. Section 6. Make corrections and insert words in sentence 6.

If a member of a Publication\$ or Communications Committee takes temporary leave from his duties, ...

Article XII. A new article.

\section{Communications}

The Committee to Monitor Problems in Communication shall perform such tasks in the field of communication of mathematics as are assigned to it by the Council.

Article XIII. The old Article XII should be renumbered.

Professor Anatole Beck moved that a resolution be placed on the agenda of the Annual Meeting of January 1971 for action at that 
time. The operative clause in the resolution to be considered at the January meeting was the following:

BE IT RESOLVED that the American Mathematical Society does hereby call for the prompt termination of American participation in the War in Southeast Asia, and for the withdrawal of our troops with the greatest speed possible, limited only by the availability of transport and the safety of said troops and regardless of the consequences to the present governments in Saigon and Pnom Penh.

The motion to place the resolution on the agenda failed by a vote of 18 to 23.

Professor Beck then moved that the following resolution be placed on the agenda for action at the Annual Meeting of 1971:

That the Society shall poll its membership on the question: Are you in substantial agreement or disagreement with this statement:

I favor the prompt termination of American participation in the War in Southeast Asia, and the withdrawal of our troops with the greatest speed possible, limited only by the availability of transport and the safety of said troops regardless of the consequences to the present governments in Saigon and Pnom Penh.

The resolution passed by a vote of 24 to 22 . [The Secretary wishes to emphasize that the action at the meeting of January 1971 in Atlantic City is to consist of considering and possibly deciding whether to conduct an opinion poll. He understands that if a poll is conducted, it is to be done by mail. A legal opinion and a cost estimate are to be available prior to the putative decision.]

The meeting adjourned at 4:30 P.M.

Bethlehem, Pennsylvania

Honolulu, Hawail
Everett Pitcher

Secretary

R. S. PIERCE

Associate Secretary 